\title{
Imaging the Molecular Footprints of the Heart-Brain Axis in Cardiovascular Disease
}

\author{
James T. Thackeray
}

Department of Nuclear Medicine, Hannover Medical School, Hannover, Germany

A ine of the heart, where the principal damage is incurred. However, increasingly appreciated is the fact that body systems do not operate independently, such that damage to one organ may bear grave consequences for disparate organs. The heart and brain comprise a fundamental battery for normal human health: the heart provides essential oxygen and nutrients to the central nervous system and the nervous system in turn regulates heart rate and contractility, facilitating adaptation to stress. What happens when part of this network is compromised, such as by ischemic damage? Does injury to one organ anticipate or even precipitate a detrimental response in the reciprocal organ? The interdependence of organ systems is clear, as exemplified by the influence of cancer on the cardiovascular system (1) or by systemic inflammatory diseases such as atherosclerosis (2). The emerging field of neurocardiology emphasizes the unique interaction between the heart and brain (3).

Clinical and experimental evidence suggests that myocardial ischemia imparts a higher risk of dementia, including Alzheimer disease $(4,5)$. Patients after a first myocardial infarction perform less well in cognitive testing than healthy cohorts (4). Further, retrospective studies have reported an increased incidence and risk of Alzheimer disease among patients after myocardial infarction $(5,6)$. Likewise, surgical interventions, such as coronary artery bypass, have been implicated in cognitive impairment (7). Although the precise mechanisms have not been fully elucidated, this interaction is theorized to involve a myriad of factors, including impaired cerebral blood flow, neuroinflammation, and oxidative stress.

Although thus far largely inferred from statistically significant associations of clinical findings, recent studies on experimental animals and on humans have revealed molecular footprints of such a heart-brain axis (2). The heart and brain are interconnected by inflammation via the sympathetic nervous system. Stimulation of adrenoceptors in the bone marrow niche mobilize inflammatory leukocytes, leading to local and systemic inflammation in ischemic disease $(2,8)$. The sympathetic nervous system link was extended by identification of increased ${ }^{18} \mathrm{~F}$-FDG signal in the amygdala brain region, which contributed to higher atherosclerotic plaque inflammation and incidence of major adverse cardiac events (9). Ischemic injury and consequent sympathetic activation

Received Jan. 15, 2019; revision accepted Mar. 1, 2019.

For correspondence or reprints contact: James T. Thackeray, Department of Nuclear Medicine, Hannover Medical School, Carl Neuberg Strasse 1, 30625 Hannover, Germany.

E-mail: thackeray.james@mh-hannover.de

Published online Mar. 8, 2019.

COPYRIGHT (c) 2019 by the Society of Nuclear Medicine and Molecular Imaging. DOI: 10.2967/jnumed.118.222315 can stimulate inflammatory cell release from hematopoietic reservoirs and directly impair contractile function $(3,10)$. Such studies provide a foundation to understand how the brain may influence cardiac function after ischemic damage.

To evaluate the influence of cardiac ischemic injury on the brain, we occluded a coronary artery in mice and serially assessed cardiac and neuroinflammation using PET of mitochondrial translocator protein (TSPO) as a marker of activated peripheral macrophages and brain microglia (11). At $1 \mathrm{wk}$ after myocardial infarction, mice exhibited a markedly higher TSPO PET signal emanating from the infarct territory, colocalized to infiltrating CD68-positive macrophages (Fig. 1A). After an intermediate decline to baseline levels, the TSPO PET signal was reelevated at 8 wk after myocardial infarction, localized to the remote myocardium. This TSPO upregulation was not limited to the heart. Diffuse global neuroinflammation was identified in an identical biphasic pattern, with elevated signal at $1 \mathrm{wk}$, baseline levels at $4 \mathrm{wk}$, and reelevated signal at $8 \mathrm{wk}(11)$. At both time points, TSPO colocalized to CD68-positive microglia within the cortex, suggesting cognitive consequences. The late TSPO signal was inversely proportional to cardiac function (11), and experimental heart failure has previously been shown to augment pro-inflammatory cytokine content and impair memory function (12). The biphasic neuroinflammatory response is reminiscent of Alzheimer dementia pathogenesis, for which microglial priming precedes plaque formation and neurodegeneration (13). Neuroinflammatory response was specific to cardiac injury, as lipopolysaccharideinduced quadriceps inflammation did not evoke parallel brain TSPO upregulation. Concomitant heart and brain inflammation was validated using ${ }^{11} \mathrm{C}-\mathrm{PK} 11195 \mathrm{PET}$ and multiple bed positions in myocardial infarction patients with acute ST elevation (11). Although preliminary, voxelwise statistical parametric mapping suggests involvement of the frontal cortex, consistent with murine experiments and possibly underlying cognitive effects; larger-scale studies are requisite.

Accordingly, neuroinflammation and, by extension, long-term brain function may be adversely affected by ischemic injury to the heart. The critical linking factors appear to be inflammation and sympathetic neuronal activation (Fig. 1B), both of which are targets of current and experimental medical therapy and molecular imaging agents. Refinement of novel targeted therapies requires assessment of both on-target and off-target effects, for which wholebody PET imaging may be of benefit. This is a compelling argument for the continued development of whole-body imaging (14), which may allow the impact of individual injuries to be interrogated beyond the target organ. New tracers are needed that address the direct interconnections between the heart and brain, including targeted inflammatory cell ligands and sympathetic neuronal markers $(15,16)$. 


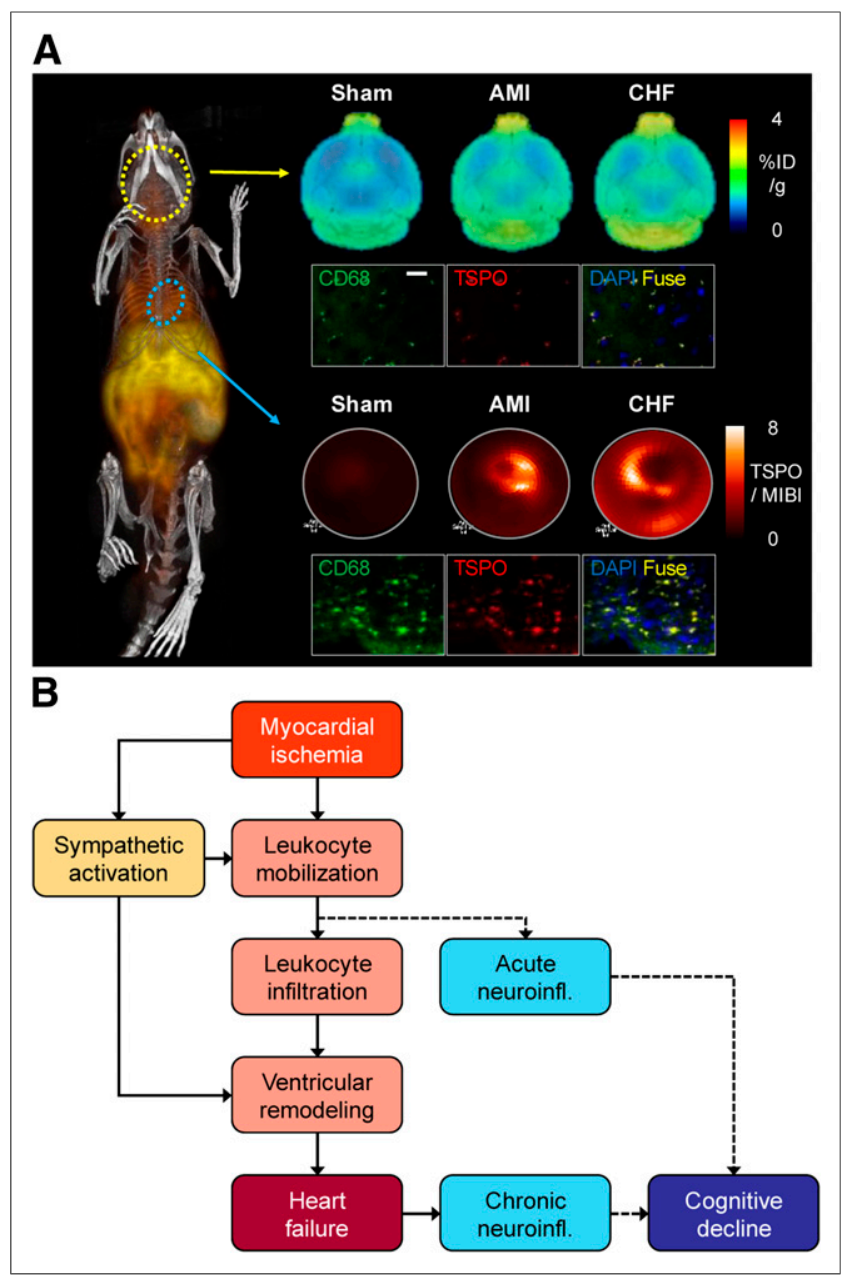

FIGURE 1. Concurrent inflammation in brain after acute myocardial infarction (AMI) and in chronic heart failure (CHF). (A) TSPO PET imaging using ${ }^{18} \mathrm{~F}-\mathrm{GE} 180$ identified cardiac and neuroinflammation in acute myocardial infarction, and recurrent neuroinflammation in heart failure.

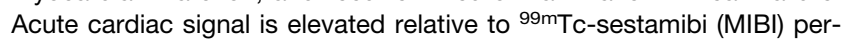
fusion in infarct territory. Increased remote myocardium TSPO signal may reflect mitochondrial dysfunction in progressive heart failure. Global neuroinflammation, particularly in frontal cortex, is observed in mouse brain early after $\mathrm{MI}$ and in CHF. Immunostaining confirms TSPO expression (red) by CD68-positive macrophages and microglia (green). (B) Schematic of heart-brain axis after myocardial infarction, where acute ischemic injury evokes local inflammation and sympathetic neuronal activation to mobilize peripheral leukocytes. This acute cardiac and neuroinflammation contributes to ventricular remodeling and may precipitate cognitive impairment. $\% \mathrm{ID} / \mathrm{g}=$ percentage injected dose per gram of tissue. DAPI = 4',6-diamidino-2-phenylindole.

The precise mechanism of this coincident inflammation remains to be determined. Neuroinflammation is a generalized response to disruption of brain homeostasis; thus, the specificity of this signal is unclear. It will be important to establish a clear connection between the initial inflammatory response after myocardial infarction and subsequent cognitive decline. Follow-up studies in animal models and patients are warranted to distinguish this interaction. Moreover, the mechanistic progression from neuroinflammation to Alzheimer disease requires further refinement. The clinical evidence and recent imaging data support an intimate network between the heart and the brain and portend the need to evaluate disease beyond the target organ.

\section{DISCLOSURE}

No potential conflict of interest relevant to this article was reported.

\section{REFERENCES}

1. Thackeray JT, Pietzsch S, Stapel B, et al. Insulin supplementation attenuates cancer-induced cardiomyopathy and slows tumor disease progression. JCI Insight. 2017;2:e93098.

2. Dutta P, Courties G, Wei Y, et al. Myocardial infarction accelerates atherosclerosis. Nature. 2012;487:325-329.

3. Chen Z, Venkat P, Seyfried D, Chopp M, Yan T, Chen J. Brain-heart interaction: cardiac complications after stroke. Circ Res. 2017;121:451-468.

4. Breteler MM, Claus JJ, Grobbee DE, Hofman A. Cardiovascular disease and distribution of cognitive function in elderly people: the Rotterdam study. BMJ. 1994;308:1604-1608.

5. Sundbøll J, Horvath-Puho E, Adelborg K, et al. Higher risk of vascular dementia in myocardial infarction survivors. Circulation. 2018;137:567-577.

6. Qiu C, Winblad B, Marengoni A, Klarin I, Fastbom J, Fratiglioni L. Heart failure and risk of dementia and Alzheimer disease: a population-based cohort study. Arch Intern Med. 2006;166:1003-1008.

7. Newman MF, Kirchner JL, Phillips-Bute B, et al. Longitudinal assessment of neurocognitive function after coronary-artery bypass surgery. $N$ Engl $\mathrm{J} \mathrm{Med}$. 2001;344:395-402.

8. Courties G, Herisson F, Sager HB, et al. Ischemic stroke activates hematopoietic bone marrow stem cells. Circ Res. 2015;116:407-417.

9. Tawakol A, Ishai A, Takx RA, et al. Relation between resting amygdalar activity and cardiovascular events: a longitudinal and cohort study. Lancet. 2017;389:834-845.

10. Min J, Farooq MU, Greenberg E, et al. Cardiac dysfunction after left permanent cerebral focal ischemia: the brain and heart connection. Stroke. 2009;40:25602563.

11. Thackeray JT, Hupe HC, Wang Y, et al. Myocardial inflammation predicts remodeling and neuroinflammation after myocardial infarction. J Am Coll Cardiol. 2018;71:263-275.

12. Meissner A, Visanji NP, Momen MA, et al. Tumor necrosis factor-alpha underlies loss of cortical dendritic spine density in a mouse model of congestive heart failure. J Am Heart Assoc. 2015;4(5):e001920.

13. Fan Z, Brooks DJ, Okello A, Edison P. An early and late peak in microglial activation in Alzheimer's disease trajectory. Brain. 2017;140:792-803.

14. Cherry SR, Jones T, Karp JS, Qi J, Moses WW, Badawi RD. Total-body PET: maximizing sensitivity to create new opportunities for clinical research and patient care. J Nucl Med. 2018;59:3-12.

15. Thackeray JT, Bengel FM. Molecular imaging of myocardial inflammation with positron emission tomography post-ischemia: a determinant of subsequent remodeling or recovery. JACC Cardiovasc Imaging. 2018;11:13401355 .

16. Thackeray JT, Bengel FM. PET imaging of the autonomic nervous system. Q J Nucl Med Mol Imaging. 2016;60:362-382. 